\title{
Формирование и исследование оптических свойств локально растянутых Ge микроструктур, встроенных в резонаторы
}

\author{
(С) Д.В. Юрасов ${ }^{1}$, Н.А. Байдакова ${ }^{1}$, В.А. Вербус ${ }^{1,2}$, Н.С. Гусев ${ }^{1}$, Е.Е. Морозова ${ }^{1}$, Д.В. Шенгуров ${ }^{1}$, \\ А.Н. Яблонский ${ }^{1}$, А.В. Новиков ${ }^{1,3}$ \\ ${ }^{1}$ Институт фозиики микроструктур Российской академии наук, \\ 603087 Нижний Новгород, Россия \\ ${ }^{2}$ Научно-исследовательский университет „Высшая школа экономики“, \\ 603155 Нижний Новгород, Россия \\ ${ }^{3}$ Нижегородский государственный университет им. Н.И. Лобачевского, \\ 603950 Нижний Новгород, Россия \\ E-mail: Inquisitor@ipmras.ru
}

Поступила в Редакцию 24 декабря 2020 г.

В окончательной редакции 30 декабря 2020 г.

Принята к публикации 30 декабря 2020 г.

\begin{abstract}
Представлены результаты формирования на SOI подложках локально растянутых Ge микроструктур (микромостиков), встроенных в резонаторы, и исследования их оптических свойств. Был рассчитан дизайн резонаторов, совместимый с геометрией локально растянутой активной области, при использовании которого, с одной стороны, наблюдалась бы эффективная локализация электромагнитного поля в активной области структуры, а с другой - минимизировалось бы влияние резонатора на величину и распределение упругих деформаций в ней. Измерения спектров микрофотолюминесценции продемонстрировали значительное возрастание интенсивности сигнала в растянутых областях Ge микроструктур по сравнению с исходной Ge пленкой. Было показано, что формирование резонаторов приводит к уменьшению деформаций в Ge микромостикаx, однако способствует увеличению интенсивности ФЛ исследуемых структур.
\end{abstract}

Ключевые слова: SiGe-структуры, деформация, растянутый $\mathrm{Ge}$, резонатор, фотолюминесценция, теплоотвод.

DOI: 10.21883/FTP.2021.05.50830.9584

\section{1. Введение}

Одной из основных проблем развития кремниевой фотоники является отсутствие эффективного КМОПсовместимого источника излучения ИК диапазона. Среди различных подходов к решению данной задачи, рассматривающихся в настоящее время, перспективным вариантом представляется использование в качестве активной среды деформированного (растянутого) Ge. Это связано с тем, что при растяжении уменьшается исходно небольшая разница между прямой и непрямой запрещенными зонами в $\mathrm{Ge}(140$ мэВ при $300 \mathrm{~K}$ в объемном $\mathrm{Ge})$. По разным оценкам, приведенным в литературе, приложение двуосных деформаций $\left(\varepsilon_{b i}\right)$ на уровне $\sim 1.5-2 \%$ или одноосных деформаций $\left(\varepsilon_{\text {uni }}\right)$ на уровне $\sim 4.7-6 \%$ способно уменьшить эту разницу до нуля, и тем самым превратить Ge в прямозонный материал $[1,2]$. Достичь таких значений деформации в сплошных Ge слоях достаточно сложно, так как для этого требуется их эпитаксиальный рост на „виртуальных подложках“ с большим параметром решетки, например, на основе буферных слоев InGaAs или GeSn. Получение таких „виртуальных подложек“ высокого кристаллического качества, вопервых, является нетривиальной задачей, а во-вторых, толщина растянутого слоя $\mathrm{Ge}$, который может быть сформирован на них без образования дефектов, ограничена критической толщиной псевдоморфного роста (обычно не более нескольких нанометров в зависимости от параметра решетки виртуальной подложки). С другой стороны, формирование сильно растянутого Ge можно обеспечить в локальных областях исходной пленки $\mathrm{Ge}$, которая при этом может быть выращена на $\mathrm{Si}$ (либо SOI) подложке. Одним из методов реализации этой идеи является так называемый „метод концентрации напряжений“ $[3,4]$. На пути достижения лазерной генерации в локально растянутых $\mathrm{Ge}$ микроструктурах, полученных данным методом, стоят две проблемы. Первая из них связана с плохим теплоотводом от свободновисящих, локально растянутых областей, формируемых методом концентрации напряжений $[5,6]$. Это существенно ограничивает диапазон мощностей накачки для таких структур. Вторая проблема - это создание резонаторов в локально растянутых Ge микроструктурах, которые не оказывали бы существенного влияния на распределение деформации в них. Встраивание свободновисящих, растянутых Ge-микроструктур в резонатор позволило продемонстрировать в таких микроструктурах лазерную генерацию при низких температурах [7]. Однако из-за плохого теплоотвода от свободновисящей активной области генерация была продемонстрирована только при возбуждении импульсным излучением с малой длительностью импульса и при низких температурах [7]. Для решения проблемы теплоотвода от активной области структуры могут быть использованы методы сращива- 
ния [6] или адгезии локально растянутой области к подложке [8].

В настоящей работе теоретически и экспериментально исследована возможность встраивания в резонаторы локально растянутых $\mathrm{Ge}$ микроструктур, улучшение теплоотвода в которых достигнуто за счет адгезии активной области к подложке за счет действия капиллярных сил [9]. Этот способ улучшения теплоотвода отличается простотой своей реализации по сравнению с подходами, использующими методы сращивания. Было исследовано влияние резонаторов на излучательные свойства и распределение упругих напряжений в локально деформированных Ge микроструктурах.

\section{2. Методика эксперимента}

Исходный сплошной слой Ge был выращен на подложке SOI методом молекулярно-пучковой эпитаксии. Отметим, что в предыдущей работе авторов [8] для реализации адгезии локально растянутых Ge структур использовались подложки SOI с тонкими слоями верхнего $\mathrm{Si}$ и $\mathrm{SiO}_{2}$ (100 и 200 нм соответственно). В настоящей работе было показано, что адгезия активной области структуры к нижележащим слоям подложки возможна и для Ge слоев, выращенных на „стандартных“ SOI подложках с более толстым ( 250 нм) приборным слоем Si. Толщина скрытого слоя окисла в используемых в настоящей работе SOI подложках составляла 3 мкм. Большая толщина слоя скрытого окисла позволяла обеспечить ограничение распространения излучения в вертикальном направлении. Для снижения плотности дефектов в активной области, в качестве которой служил $n$-Ge слой, легированный $\mathrm{Sb}$, его рост осуществлялся на $\mathrm{Ge} / \mathrm{SOI}$ буфере (толщина $\mathrm{Ge} \sim 600 \mathrm{HM}$ ), полученном с помощью метода „двухтемпературного роста“ с последующим циклическим отжигом в вакуумной камере [10-12]. Толщина $n$-Ge слоя составляла 300 нм, а концентрация электронов в нем $\sim 3 \cdot 10^{19} \mathrm{~cm}^{-3}$. Использование легирования донорами имело цель повысить эффективность излучательной рекомбинации в $\mathrm{Ge}$, что, как было показано ранее, возможно при оптимально подобранном уровне легирования [13-17]. Для легирования Ge сурьмой использовался ранее разработанный авторами метод, описанный в [18]. В выращенной сплошной пленке $\mathrm{Ge}$ из-за разницы коэффициентов температурного расширения Ge и $\mathrm{Si}$ наблюдалась деформация растяжения на уровне $0.2-0.25 \%$.

Создание локально-деформированных Ge микроструктур в геометрии типа „микромостик“ осуществлялось с помощью лазерной литографии, плазмохимического и „Влажного“ селективного травления. Подробно процесс формирования локально растянутых $\mathrm{Ge}$ микромостиков с адгезией к подложке описан в [8]. В настоящей работе дизайн микромостиков был изменен путем добавления с обеих сторон от мостика вытравленных областей, геометрически представляющих собой кольцевые секто- ра со сглаженными углами (рис. $1, a$ ). Данные сектора представляли собой фрагменты сферического зеркала, которые и образовывали резонатор. В результате формирования такого резонатора создавалось ограничение для распространения излучения в направлении вдоль мостика (в двух других направлениях оно обеспечивалось ограниченными геометрическими размерами самого мостика). Для выявления влияния резонатора на оптические свойства Ge микромостиков на той же структуре были сформированы микромостики без резонатора и микромостики, содержащие сегмент сферического зеркала лишь с одной стороны.

Как указывалось выше, в настоящей работе использовалась SOI подложка с более толстым слоем $\mathrm{Si}$, отделяющим слой $\mathrm{Ge}$ от скрытого слоя окисла, чем в предыдущей работе авторов, в которой была реализована адгезия микромостиков к слою окисла [8]. В связи с этим в настоящей работе вместо изопропилового спирта, использовавшегося для реализации адгезии в [8], была использована вода, поскольку она обладает большим коэффициентом поверхностного натяжения. При испарении слоя воды за счет адгезии возникал механический контакт между мостиком и нижележащим слоем $\mathrm{SiO}_{2}$.

Расчеты дизайна резонаторов, учитывающие распределение электромагнитного поля и упругих напряжений в активной области структуры, были выполнены с помощью метода конечных элементов с использованием программного пакета Comsol Multiphysics. Экспериментальные исследования полученных $\mathrm{Ge}$ микроструктур проводились с помощью сканирующей электронной микроскопии (СЭМ), интерферометрии белого света и спектроскопии микрофотолюминесценции при комнатной температуре (микроФЛ). При исследовании микроФЛ структуры возбуждались импульсным излучением $\mathrm{Nd}$ :YAG-лазера (длина волны $532 \mathrm{Hм}$, частота повторения 80 МГц, длительность импульса $\sim 10$ пс), которое фокусировалось на образец с помощью объектива $\times 10$ (диаметр пятна 2-3 мкм). Этот же объектив использовался для сбора излучения исследуемых микроструктур. Регистрация спектров микроФЛ осуществлялась с помощью решеточного монохроматора Acton 2300i и многоканального детектора OMA-V на основе линейки фотодиодов InGaAs.

\section{3. Результаты и их обсуждение}

В настоящей работе были сформированы микромостики, созданные с использованием вышеописанной схемы с адгезией к нижележащим слоям SOI подложки. Дизайн мостика с резонатором, рассмотренный в настоящей работе, показан схематически на рис. $1, a$, а его СЭМ-снимок - на рис. $1, b$. В методе „концентрации напряжений“, использованном для формирования микромостиков, величина упругих напряжений в области мостика зависит от геометрических параметров струк- 


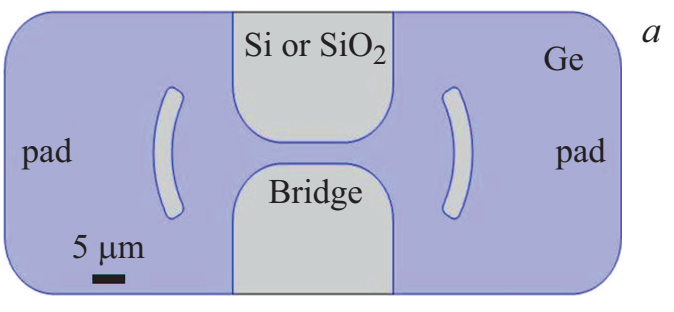

$a$
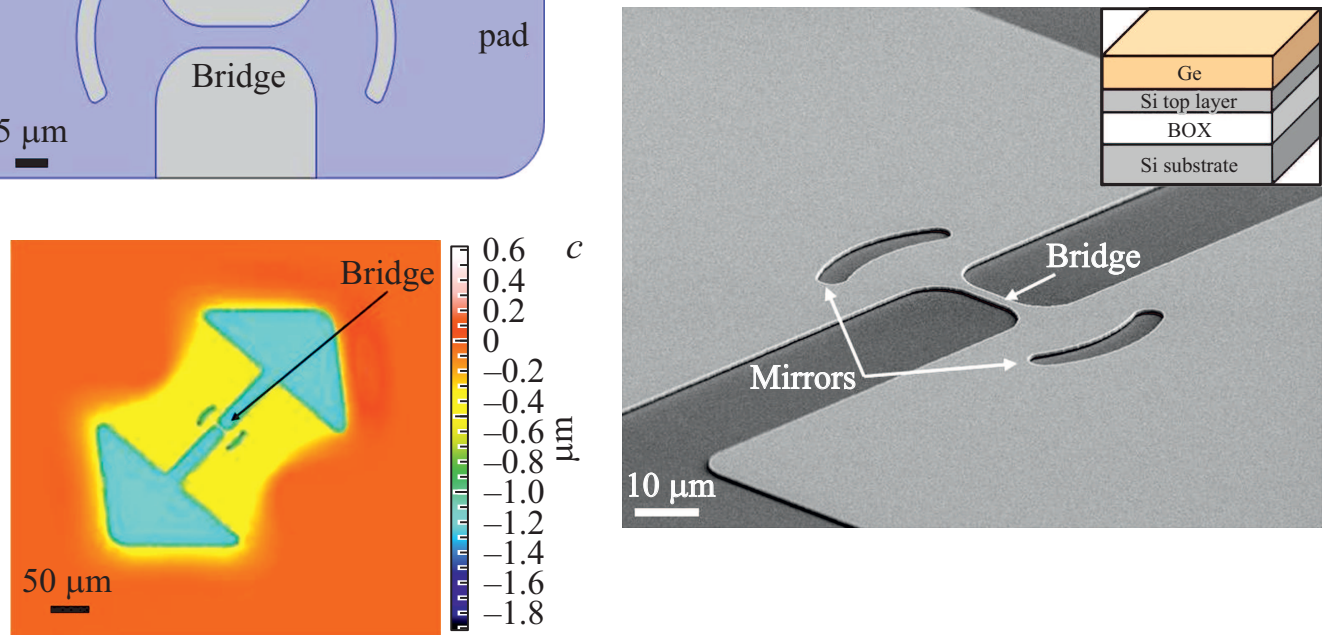

Рис. 1. $a$ - схема микромостика („bridge“) с резонатором, сформированным за счет вытравливания в „берегах“ („рад““) кольцевых секторов по обе стороны от мостика. $b-$ СЭМ-снимок сформированного мостика. На вставке схематически представлено изображение поперечного разреза исходной структуры. $c$ - изображение мостика, полученное с помощью интерферометрии белого света. Цветовой шкалой указан масштаб высот. (Цветной вариант рисунка представлен в электронной версии статьи).

туры (и упругих констант материалов). Более подробное описание геометрических размеров структур приведено в более ранней работе авторов [19]. В настоящей работе были сформированы мостики со схожими параметрами, а основным отличием было наличие (или отсутствие) резонатора.

Дизайн резонатора (рис. 1) был выбран таким образом, чтобы максимально увеличить коэффициент излучения в диапазоне длин волн 1.5-2 мкм, в котором наблюдается сигнал люминесценции от растянутого $\mathrm{Ge}$, а также обеспечить фокусировку отраженного от зеркал излучения обратно в микромостик (рис. 2). Численные расчеты собственных мод в рассматриваемом диапазоне длин волн для данной структуры были выполнены двумерным методом конечных элементов с помощью RF модуля в пакете Comsol Multiphysics. Один из характерных результатов представлен на рис. 2 .

На рис. 2 видно сужение волнового фронта в районе центра микромостика, где наблюдается максимальная интенсивность поля, т.е. реализуется эффективная „фокусировка" отраженного излучения обратно в активную область структуры. Также можно отметить, что в вытравленных областях, формирующих зеркала, в рассматриваемом диапазоне длин волн образуется стоячая волна, что позволяет этим областям достаточно эффективно работать в качестве отражающих зеркал.

Несмотря на относительно небольшие размеры вытравленных областей, формирующих резонатор, они находятся вблизи активной области (микромостика), и удаление части материала берегов для формирования резонатора может оказывать влияние на распределение упругих напряжений в структуре. В настоящей работе

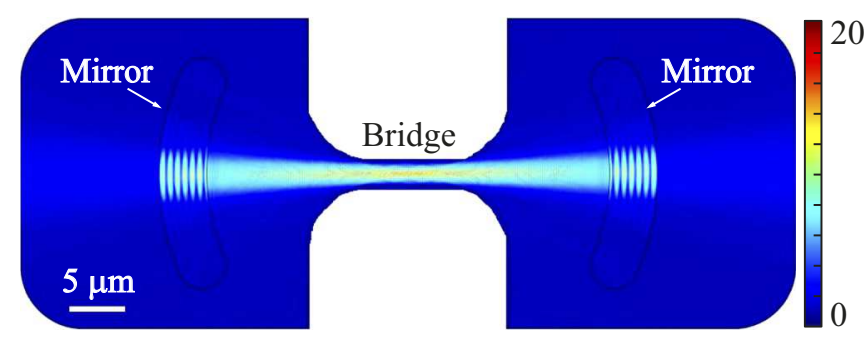

Рис. 2. Результат расчета пространственного распределения поля в образце (для $\lambda=1.9$ мкм). Цветовой шкалой показана величина модуля электрического поля. Длина мостика 10 мкм.

при оптимизации дизайна резонаторов его геометрические параметры подбирались так, чтобы, по возможности, минимизировать снижение величины упругих напряжений в активной области в целом и при этом добиться максимально однородного их распределения в микромостике. На рис. 3 показан результат теоретического расчета распределения упругих напряжений для одной из исследованных структур (длина мостика 5 мкм, ширина - 1.8 мкм), выполненный с помощью метода конечных элементов. Согласно расчетам, в структурах с микромостиками в зависимости от наличия и количества зеркал резонатора величина деформации $\varepsilon$ составляет от 2.5 до 4.2\% ( $\pm 0.35 \%$ - погрешность, связанная в основном с неточностью определения исходной деформации в сплошной пленке $\mathrm{Ge}$ до формирования микромостиков) (рис. 3, b).

Из расчетов распределения деформации следует, что вытравливание зеркал, формирующих резонатор, замет- 

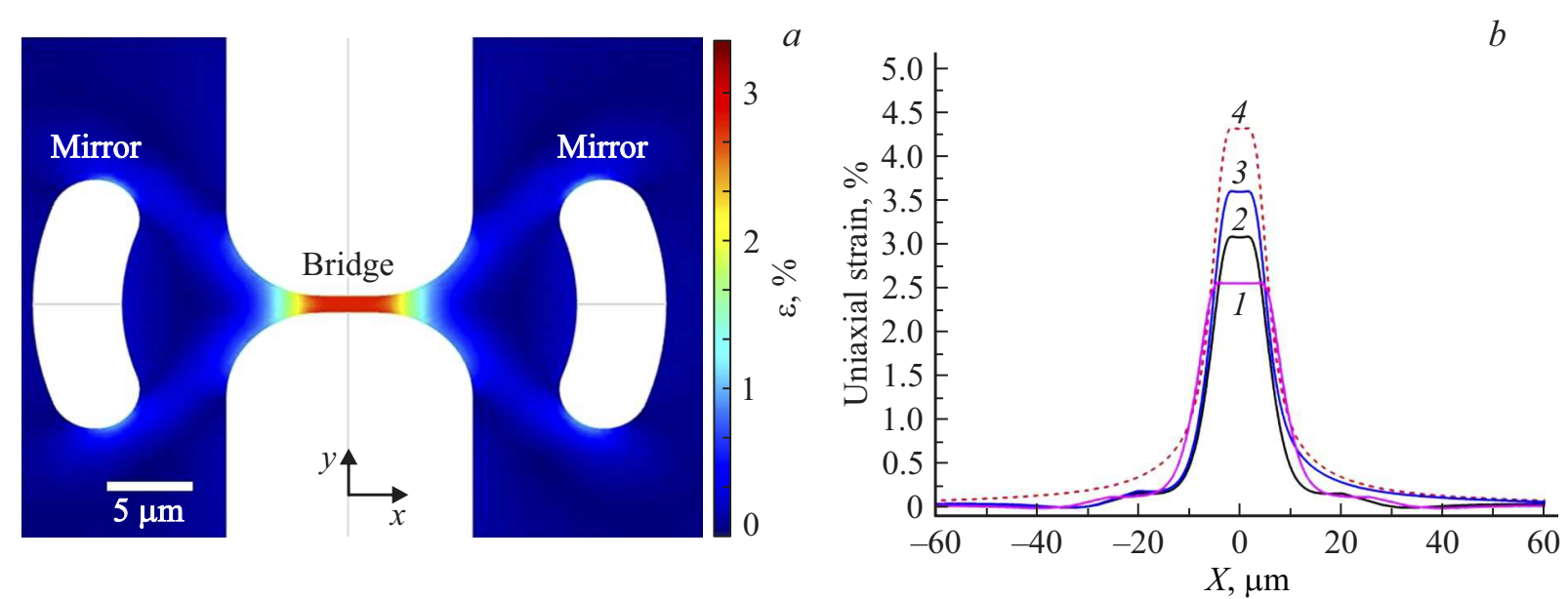

Рис. 3. $a$ - результаты расчета деформаций в микромостике длиной 5 мкм, встроенном в резонатор, и его окружении. Цветовой шкалой показана величина растяжения вдоль оси $X . b$ - расчетные кривые распределения $\varepsilon_{x x}$ компоненты тензора деформаций для микромостиков со следующими параметрами: 1 - мостик длиной 10 мкм с двумя „зеркалами“, 2 - мостик длиной 5 мкм с двумя „зеркалами“, 3 - мостик длиной 5 мкм с одним „зеркалом“, 4 - мостик длиной 5 мкм без „зеркал“.

но снижает величину максимальной деформации в микромостиках. С другой стороны, экспериментально было обнаружено, что в используемых структурах происходит разрушение микромостиков, для которых расчеты распределения деформации дают величину растяжения в них более $3.5 \%$. В мостиках с двумя зеркалами этого предельного значения деформации можно достичь за счет соответствующего выбора размеров мостика и берегов. По мнению авторов, достичь более высоких деформаций растяжения в мостиках возможно путем повышения кристаллического качества исходных структур (уменьшение плотности дефектов, снижающих механическую прочность материала), а также, потенциально, за счет оптимизации процессов литографии/травления.

Из-за особенностей геометрии микромостиков из их СЭМ-изображений невозможно однозначно судить о наличии либо отсутствии механического контакта мостика с нижележащим слоем (рис. $1, b)$. Для ответа на данный вопрос были проведены исследования с помощью интерферометрии белого света, которая позволяет определять перепад высот между отдельными областями структуры с точностью до единиц нанометров. Снимок мостика с двумя зеркалами, полученный с помощью этого метода, показан на рис. 1,c. На приведенном снимке легко можно видеть, что область берегов и мостика (желтый цвет) находится на другой высоте, по сравнению с непроцессированной областью (оранжевый цвет, соответствующий нулевому уровню отчета высоты (см. цветовую шкалу на рис. $1, c)$ ). Измеренная разность высот между областью мостика и берегов, по сравнению с непроцессированной областью, хорошо соответствует толщине вытравленного из-под мостика слоя Si. Это является подтверждением того, что для исследованных мостиков, сформированных на SOI подложке с толщиной $\mathrm{Si}$-слоя над окислом более 250 нм, была реализована адгезия к слою захороненного окисла. Таким образом, были сформированы локально растянутые Ge микромостики с различным уровнем деформации, приведенные в механический контакт с нижележащим слоем $\mathrm{SiO}_{2}$, часть из которых была встроена в резонатор вышеописанного дизайна.

Исследование оптических свойств изготовленных структур было проведено методом спектроскопии микроФЛ при $300 \mathrm{~K}$. На рис. 4 приведены спектры микроФЛ исходной $n$-Ge/SOI структуры (кривая 1 ) и двух сформированных на ней микромостиков после разных этапов их создания. Спектр 2 на рис. 4 получен для мостика после проведения плазмохимического травления, но до операции селективного удаления слоя $\mathrm{Si}$ между слоем $\mathrm{Ge}$ и захороненным слоем окисла (далее „неподвешенный мостик“). В мостиках, не подвергнутых процедуре „подвешивания“, не происходит заметного перераспределения упругих деформаций между самим мостиком и берегами, т.е. деформация таких мостиков остается на уровне деформации исходной пленки Ge. Спектр 3 на рис. 4 получен для мостика, прошедшего все этапы формирования, включая селективное удаление слоя $\mathrm{Si}$ между слоем $\mathrm{Ge}$ и захороненным слоем $\mathrm{SiO}_{2}$, a также адгезию к нижележащим слоям подложки (далее „Подвешенный мостик“).

Как видно из рис. 4, вид и положение спектра ФЛ неподвешенного мостика повторяет вид спектра ФЛ исходной структуры, что указывает на близкий уровень деформации в неподвешенном мостике и исходном $n$-Ge-слое. Однако интенсивность сигнала ФЛ от неподвешенного мостика в $\sim 1.5$ раза выше, чем от исходной структуры. Наблюдаемое увеличение интенсивности ФЛ неподвешенного мостика по сравнению с исходным слоем Ge связывается с лучшим выводом излучения из мостика через его боковые поверхности.

Значительное увеличение деформации растяжения в подвешенном мостике за счет перераспределения де- 


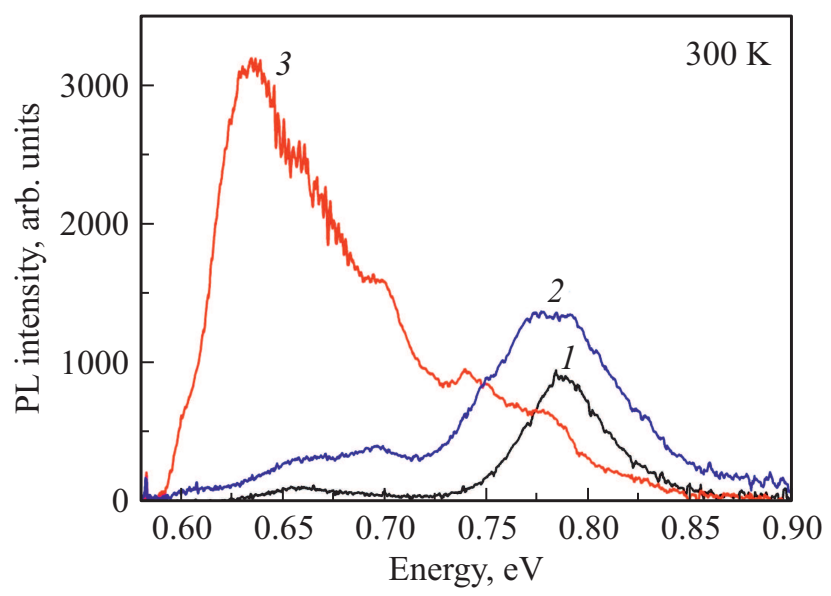

Pис. 4. Спектры микроФЛ: исходной структуры с $n$-Ge/SOI слоем (1), не подвешенного мостика (2) и подвешенного мостика (3). Температура измерения $300 \mathrm{~K}$.

формации между ним и берегами приводит к увеличению интенсивности его сигнала ФЛ в $\sim 3.5$ раза в максимуме и приблизительно на порядок в интеграле по сравнению с исходной структурой. На значительный рост деформации растяжения в подвешенном мостике указывает смещение положения максимума его сигнала ФЛ в область меньших энергий, что соответствует теоретическим представлениям об изменении зонной структуры Ge при растяжении [2]. Таким образом, из представленных на рис. 4 спектров микроФЛ можно сделать вывод, что основной вклад в увеличение интенсивности сигнала ФЛ в растянутом мостике вносит рост деформации растяжения в нем и связанное с этим уменьшение энергетического зазора между прямой и непрямой запрещенными зонами в растянутом Ge. Наблюдаемое положение и форма спектра ФЛ микромостиков хорошо описывается расчетами оптических переходов из Г-долины зоны проводимости в зоны легких и тяжелых дырок при использовании рассчитанных значений деформации мостиков и учета расщепления подзон легких и тяжелых дырок в результате одноосной деформации. Детальный теоретический анализ сигнала ФЛ исследованных $\mathrm{Ge}$ микромостиков будет представлен в отдельной работе.

Для экспериментальной оценки влияния сформированного резонатора на люминесценцию $\mathrm{Ge}$ микромостика были исследованы спектры микроФЛ нескольких растянутых микромостиков с одинаковыми геометрическими параметрами, отличавшихся только наличием/отсутствием резонатора. Исследовались две группы мостиков, имевших длины 5 и 10 мкм. Как и ожидалось из теоретических оценок упругих напряжений в микромостиках (рис. $3, b$ ), отсутствие одного или обоих зеркал резонатора приводит к смещению спектров ФЛ в более низкоэнергетическую область (рис. 5), что вызвано увеличением одноосной деформации в мостиках. Несмотря на уменьшение деформации растяжения, интенсив- ность сигнала ФЛ от микромостиков с резонаторами в несколько раз выше, чем от мостиков без них (см. спектры 2 и 3 для мостиков длиной 5 мкм и спектры 4 и 5 для мостиков длиной 10 мкм на рис. 5). При этом наибольшая интенсивность ФЛ наблюдается для мостиков с двумя зеркалами. Корректное количественное сравнение интегральных интенсивностей сигнала ФЛ различных мостиков затруднено в связи с тем, что значительная часть излучения мостиков без резонатора, характеризующихся наибольшей деформацией растяжения, расположена вне рабочего диапазона используемого фотоприемника InGaAs (рис. 5).

Для мостиков с двумя зеркалами, т.е. с полностью сформированным резонатором (спектры 2 и 4 на рис. 5), наряду с наибольшей интенсивностью ФЛ наблюдается модуляция интенсивности в спектре ФЛ. Данная модуляция связывается с проявлением резонансов Фабри-Перо. Подтверждением этого является наличие данной модуляции только в мостиках с двумя зеркалами, а также изменение периода этой модуляции при изменении расстояния между зеркалами для мостиков различной длины. Расстояние между соседними максимумами в наблюдаемой интерференционной картине для различного расстояния между зеркалами (для мостиков разной длины) хорошо совпадает с рассчитанным расстоянием между соседними резонансами Фабри-Перо. Подобные модуляции сигнала ФЛ для микромостиков, встроенных в резонаторы различного вида, также наблюдались другими авторами [5-7], что подтверждает эффективность резонаторов, сформированных в настоящей работе (с точки зрения оптического ограничения в направлении вдоль мостика). Данный элемент является необходимым условием для наблюдения лазерного эффекта в исследуемых структурах.

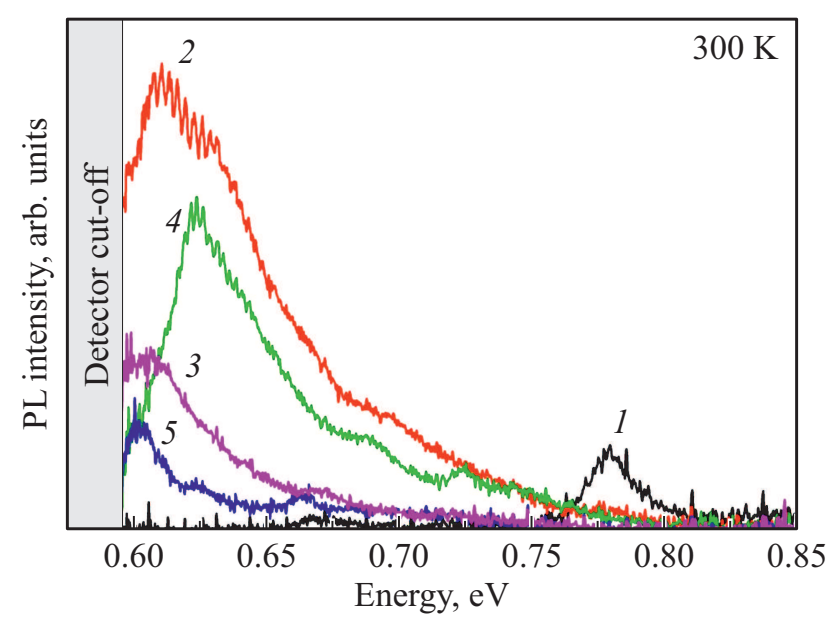

Рис. 5. Спектры микроФЛ исходного сплошного $\mathrm{Ge}$ слоя (1), мостиков с двумя зеркалами длиной 5 мкм (2) и 10 мкм (4), мостика длиной 5 мкм с одним зеркалом (3) и мостика длиной 10 мкм без зеркал (5). Серым показана зона вне рабочего спектрального диапазона детектора. Температура измерения $300 \mathrm{~K}$. 
В настоящей работе все оптические измерения были проведены при $300 \mathrm{~K}$, и стимулированного излучения от растянутого Ge достичь не удалось. В литературе было показано, что одним из факторов, затрудняющих реализацию стимулированного излучения в таком материале при комнатной температуре, является межподзонное поглощение в валентной зоне (,inter-valence band absorption“, IVBA) [6,20,21]. При понижении температуры данный негативный фактор ослабевает [22], что снижает общие потери. Именно с этим фактором авторы ряда работ связывали наблюдение лазерной генерации в растянутом Ge только при криогенных температурах [6,7,23]. В связи с этим представляется перспективным проведение низкотемпературных люминесцентных измерений в сформированных образцах с целью изучения возможности достижения в них условий для реализации стимулированного излучения.

\section{4. Заключение}

В работе представлены результаты по формированию локально растянутых $\mathrm{Ge}$ микромостиков, встроенных в резонаторы, и исследованию их оптических свойств. Были рассчитаны параметры резонаторов, полученных путем формирования пары фрагментов сферических зеркал по обе стороны от мостика, совместимых с дизайном активной, локально растянутой области. Были сформированы микромостики с адгезией к нижележащим слоям подложки, что позволило значительно улучшить отвод тепла от активной области. Продемонстрировано, что, несмотря на уменьшение деформации растяжения в Ge микромостике при формировании одного или двух зеркал, встраивание мостика в резонатор значительно увеличивает интенсивность его ФЛ. В спектрах ФЛ мостиков, встроенных в резонаторы, наблюдалась характерная модуляция сигнала ФЛ, связываемая с проявлением резонансов Фабри-Перо.

Таким образом, результаты исследований оптических свойств локально растянутых Ge структур в совокупности с возможностью реализации эффективного отвода тепла от них за счет обеспечения их контакта с подложкой свидетельствуют о перспективности таких структур для создания на их основе $\mathrm{Si}$-совместимых излучателей ближнего ИК-диапазона.

\section{Финансирование работы}

Работа выполнена при финансовой поддержке Российского научного фонда (грант № 19-72-10011) с использованием оборудования Центра коллективного пользования „Физика и технология микро- и наноструктур“ ИФМ PAH.

\section{Конфликт интересов}

Авторы заявляют, что у них нет конфликта интересов.

\section{Список литературы}

[1] C. Boztug, J.R. Sanchez-Perez, F. Cavallo, M.G. Lagally, R. Paiella. ACS Nano, 8, 3136 (2014).

[2] R. Geiger, T. Zabel, H. Sigg. Front. Mater., 2, 52 (2015).

[3] R.A. Minamisawa, M.J. Süess, R. Spolenak, J. Faist, C. David, J. Gobrecht, K.K. Bourdelle, H. Sigg. Nature Commun., 3, 1096 (2012).

[4] M.J. Süess, R. Geiger, R.A. Minamisawa, G. Schiefler, J. Frigerio, D. Chrastina, G. Isella, R. Spolenak, J. Faist, H. Sigg. Nature Photonics, 7, 466 (2013).

[5] J. Petykiewicz, D. Nam, D.S. Sukhdeo, S. Gupta, S. Buckley, A.Y. Piggott, J. Vuckovic, K.C. Saraswat. Nano Lett., 16, 2168 (2016).

[6] S. Bao, D. Kim, C. Onwukaeme, S. Gupta, K. Saraswat, K.H. Lee, Y. Kim, D. Min, Y. Jung, H. Qiu, H. Wang, E.A. Fitzgerald, C.S. Tan, D. Nam. Nature Commun., 8, 1845 (2017).

[7] F.T. Armand Pilon, A. Lyasota, Y.-M. Niquet, V. Reboud, V. Calvo, N. Pauc, J. Widiez, C. Bonzon, J.-M. Hartmann, A. Chelnokov, J. Faist, H. Sigg, Nature Commun., 10, 2724 (2019).

[8] Д.В. Юрасов, Н.А. Байдакова, В.А. Вербус, Н.С. Гусев, А.И. Машин, Е.Е. Морозова, А.В. Нежданов, А.В. Новиков, Е.В. Скороходов, Д.В. Шенгуров, А.Н. Яблонский. ФТП, 53, 1360 (2019).

[9] N. Tas, T. Sonnenberg, H. Jansen, R. Legtenberg, M. Elwenspoek. J. Micromech. Microeng., 6, 385 (1996).

[10] H.-C. Luan, D.R. Lim, K.K. Lee, K.M. Chen, J.G. Sandland, K. Wada, L.C. Kimerling. Appl. Phys. Lett., 75, 2909 (1999).

[11] J.-M. Hartmann, A. Abbadie, J.P. Barnes, J.M. Fedeli, T. Billon, L. Vivien. J. Cryst. Growth, 312, 532 (2010).

[12] Д.В. Юрасов, А.И. Бобров, В.М. Данильцев, А.В. Новиков, Д.А. Павлов, Е.В. Скороходов, М.В. Шалеев, П.А. Юнин. ФТП, 49, 1463 (2015).

[13] J. Liu, X. Sun, D. Pan, X. Wang, L.C. Kimerling, T.L. Koch, J. Michel. Opt. Express, 15, 11272 (2007).

[14] B. Schwartz, A. Klossek, M. Kittler, M. Oehme, E. Kasper, J. Schulze. Phys. Status Solidi C, 11, 1686 (2014).

[15] M.R. Barget, M. Virgilio, G. Capellini, Y. Yamamoto, T. Schroeder. J. Appl. Phys., 121, 245701 (2017).

[16] D.V. Yurasov, A.V. Antonov, M.N. Drozdov, P.A. Yunin, B.A. Andreev, P.A. Bushuykin, N.A. Baydakova, A.V. Novikov. J. Cryst. Growth, 491, 26 (2018).

[17] D.V. Yurasov, A.V. Novikov, N.A. Baidakova, E.E. Morozova, P.A. Yunin, D.V. Shengurov, A.V. Antonov, M.N. Drozdov, Z.F. Krasilnik. Semicond. Sci. Technol., 33, 124019 (2018).

[18] D.V. Yurasov, A.V. Antonov, M.N. Drozdov, V.B. Schmagin, K.E. Spirin, A.V. Novikov. J. Appl. Phys., 118, 145701 (2015).

[19] А.В. Новиков, Д.В. Юрасов, Е.Е. Морозова, Е.В. Скороходов, В.А. Вербус, А.Н. Яблонский, Н.А. Байдакова, Н.С. Гусев, К.Е. Кудрявцев, А.В. Нежданов, А.И. Машин. ФТП, 52, 1331 (2018).

[20] L. Carroll, P. Friedli, S. Neuenschwander, H. Sigg, S. Cecchi, F. Isa, D. Chrastina, G. Isella, Y. Fedoryshyn, J. Faist. Phys. Rev. Lett., 109, 057402 (2012).

[21] S. Gupta, D. Nam, J. Vuckovic, K. Saraswat. Phys. Rev. B, 97, 155127 (2018).

[22] Yu.I. Morozov, L.I. Ukhanov. Sov. Phys. J., 13, 744 (1970).

[23] A. Elbaz, M. El Kurdi, A. Aassime, S. Sauvage, X. Checoury, I. Sagnes, C. Baudot, F. Boeuf, P. Boucaud. APL Photon., 3, 106102 (2018).

Редактор Г.А. Оганесян 


\section{Formation and optical properties of locally tensile strained Ge structures embedded into microcavities}

D.V. Yurasov ${ }^{1}$, N.A. Baidakova ${ }^{1}$, V.A. Verbus ${ }^{1,2}$, N.S. Gusev' ${ }^{1}$, E.E. Morozova ${ }^{1}$, D.V. Shengurov' ${ }^{1}$, A.N. Yablonskiy ${ }^{1}$, A.V. Novikiov ${ }^{1,3}$

${ }^{1}$ Institute for Physics of Microstructures, Russian Academy of Sciences, 603950 Nizhny Novgorod, Russia ${ }^{2}$ National Research University Higher School of Economics, 603155 Nizhny Novgorod, Russia ${ }^{3}$ Lobachevsky State University of Nizhny Novgorod, 603950 Nizhny Novgorod, Russia

Abstract In this work, formation of locally tensile strained Ge structures (micro-bridges) on SOI substrates embedded into microcavities is reported and their optical properties are discussed. The cavity compatible with the shape of the active region was designed in such a way as to provide an effective localization of the electromagnetic field in the active region of the structure, as well as to minimize the strain redistribution due to the cavity formation. Microphotoluminescence (PL) studies have shown a remarkable enhancement of the PL intensity for the locally strained areas as compared to the initial Ge film. It was shown that the formation of a microcavity leads to a decrease in the maximum strain in the active region of the structure, but provides an overall increase in the PL intensity. 